\title{
Reconstruction on fast neutron CT for concrete structure inspection with a pixel-type detector by applying linear scanning method
}

\author{
Mingfei Yan ${ }^{1, *}$, Yasuo Wakabayashi ${ }^{1}$, Yoshie Otake ${ }^{1,2}$, Yujiro Ikeda $^{1,3}$, Atsushi Taketani ${ }^{1}$, Takao Hashiguchi ${ }^{1}$, Sheng \\ Wang $^{2,1}$, Binbin Tian ${ }^{2}$, Takaoki Takanashi ${ }^{1}$, Tomohiro Kobayashi ${ }^{1}$, and Baolong Ma ${ }^{2,1}$ \\ ${ }^{1}$ RIKEN center for advanced photonics, RIKEN, Wako, Saitama, Japan \\ ${ }^{2}$ School of Energy and Power Engineering, Xi'an Jiaotong University, Xi'an, China \\ ${ }^{3}$ J-PARC Centre, Japan Atomic Energy Agency, Naka-gun, Ibaraki, Japan
}

\begin{abstract}
Concrete structure has been widely used in bridges and highways, however, its performance will be deteriorated after long term serving or suffering disaster. Since fast neutron has strong transmission ability and is sensitive to water content in the concrete structure, it can provide an effective probe to inspect the inner structure of concrete with non-destructive way. Thus, we propose a fast neutron imaging and reconstruction system of 3D CT for concrete structure inspection with Riken accelerator-driven compact neutron source (RANS) using a fast neutron pixel-type detector, which has $8 \times 8$ pixels. To have a good space resolution on the reconstructed image, a rotation + linear scanning method is devised and is used to collect the projection data from experiment or calculation. In this paper, reconstruction for a concrete object containing both iron bars and acrylic bars for simulation of water has been conducted. As a result, 3D image of $1 \mathrm{~cm}$ diameter bar is reconstructed by the sparse reconstruction algorithm.
\end{abstract}

\section{Introduction}

Concrete structure has been widely used in the modern buildings (such as bridges, high ways and so on). It is important to inspect the health condition of the concrete structure, especially when the buildings are in service for a long time or have suffered from natural disaster (like earthquake). Usually, the technologies suitable for non-destructive inspection for concrete structure include ground penetrating radar method, ultrasonic wave method, radiation method (X-ray and neutron) and so on ${ }^{[1]}$. In the radiation method, radiation imaging can directly provide the radiography and tomography images for concrete structure. At present, the X-ray imaging has been widely used in situ non-destructive inspection. Compared with $\mathrm{x}$-ray, neutron has strong transmission ability in medium, and is sensitive to water content, therefore, it can show evident advantages in concrete sutrcture inspection. However, neutron imaging is still under developing ${ }^{[2-3]}$. In addition, because the crosstalk phenomenon is serious in fast neutron imaging detector, its space resolution is not good as thermal neutron detector, which ranges from $\sim \mathrm{mm}$ to $\sim \mathrm{cm}^{[4]}$. If better space resolution in fast neutron imaging is expected, special method for collecting images should be employed. Thus we have started to develop a fast neutron imaging and reconstruction system of 3D CT for concrete structure inspection in situ with compact neutron source.

Basically, the algorithms involving CT image reconstruction can be divided into analytic and iterative classes. The famous analytic algorithm is filter back projection (FBP) algorithm, which is widely used in medical and industrial $\mathrm{CT}$ at present, and its reconstruction results is regarded as the golden standard. However, when the number of projection data is not enough, especially in sparse sampling case, a large number of artifacts will appear on the reconstructed images. However, reconstruction with iterative class algorithms usually can get better results in this case. As a kind of iterative class algorithm, the sparse reconstruction algorithm ${ }^{[5]}$ utilizes the sparse characteristic of the image, which can significantly decrease the experiment time to collect the projection data.

\section{Compact neutron source and fast neutron pixel-type detector}

RIKEN accelerator-driven compact neutron source (RANS) was developed in 2013, which utilizes $7 \mathrm{MeV}$

* Corresponding author: mingfei.yan@riken.jp 
proton to bombard $300 \mu \mathrm{m}$ thickness beryllium target to produce neutron. As is shown in figure 1, the main components of RANS includes proton accelerator section and target station section. The accelerator section mainly consists of ion source, radio-frequency quadrupole (RFQ) and drift-tube-linacs (DTL). The target station mainly contains beryllium target, cooling water, polyethylene moderator, graphite reflector and layered shieling materials. The moderator can be removed from the side of the target station when implementing fast neutron experiment.

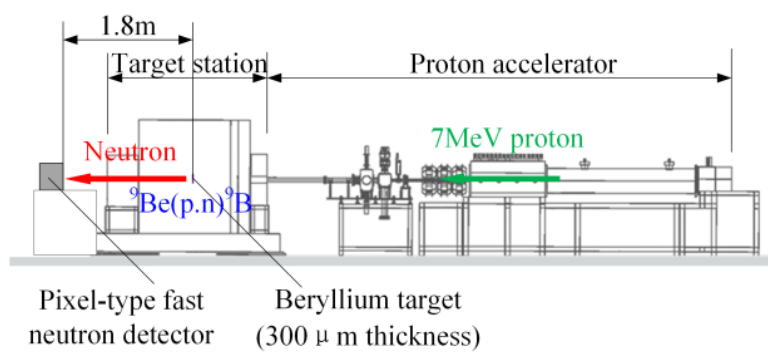

Fig. 1. Structure diagram of RANS.

The maximum current of RANS is $100 \mu \mathrm{A}$, and the corresponding energy spectrum at the location of $1.8 \mathrm{~m}$ far away from the target is shown in figure 2 , which is simulated by Geant $4 \operatorname{code}^{[6]}$. In the simulation, a neutron generation function is employed to calculate the neutron spectrum produced from the ${ }^{9} \mathrm{Be}+\mathrm{p}$ reaction with $7 \mathrm{MeV}$ protons $^{[7]}$. The peak intensity of fast neutron is nearby $10^{6} \mathrm{~cm}^{-2} \cdot \mathrm{s}^{-1}$ at the location of $1.8 \mathrm{~m}$ far away from the target.

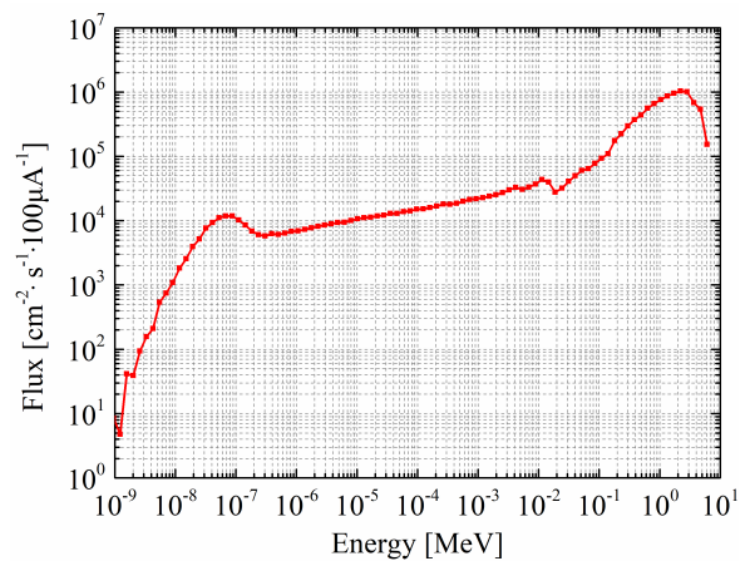

Fig. 2. Energy spectrum at $1.8 \mathrm{~m}$ far away from the target (without moderator).

To achieve non-destructive inspection for concrete structure by fast neutron imaging method, our team has developed a fast neutron pixel type detector (shown in figure 3 ), which has $8 \times 8$ pixels, and the size of each pixel is $2 \mathrm{~cm} \times 2 \mathrm{~cm} \times 5 \mathrm{~cm}$ (thickness). It utilizes the BC408 plastic scintillator as the neutron to recoiled proton convertor and scintillator, multiple-pixel photon counter (MPPC) as the light to electric signal convertor. As the electric signal produces, the number of counts can be recorded by the counter after passing though the amplifier and comparator. The detector is triggered by the accelerator signal, which is helpful for eliminating the counts produced by lower energy neutron.
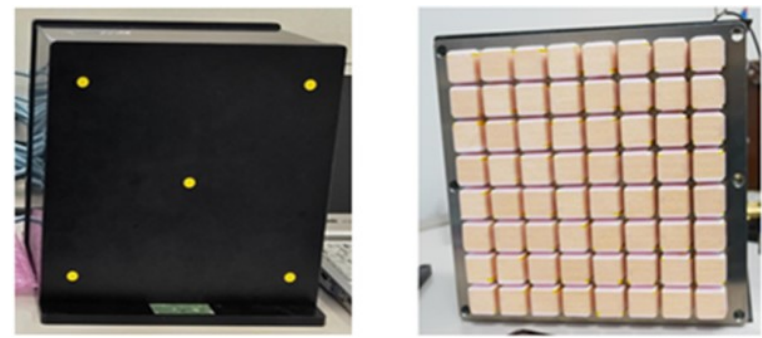

Fig. 3. Fast neutron pixel-type detector

\section{Principle of CT and image reconstruction}

Neutron intensity attenuates in the matter as LambertBeer law:

$$
I(x, y)=I_{0}(x, y) e^{-\int_{L} \mu(x, y) d l}
$$

where $I_{0}(x, y)$ and $I(x, y)$ refer to the incident and transmitted intensities respectively; $\mu(x, y)$ denotes the macroscopic neutron cross section of the matter. The principle of CT image reconstruction is calculating $\mu(x, y)$ from $I_{0}(x, y), I(x, y)$ and the geometry configuration of CT system.

CT image reconstruction with iterative class algorithms can be regarded as solving the linear equation set:

$$
\begin{aligned}
& \mathbf{P}_{M \times 1}=\mathbf{W}_{M \times N} \mathbf{U}_{N \times 1} \\
& \text { i.e. }\left[\begin{array}{c}
p_{1} \\
p_{2} \\
\vdots \\
p_{M}
\end{array}\right]=\left[\begin{array}{ccccc}
w_{11} & w_{12} & \cdots & & \\
w_{21} & w_{22} & \cdots & & \\
& \vdots & \ddots & \vdots & \\
& & & \\
w_{M 1} & w_{M 2} & \cdots & \mu_{1} \\
\mu_{2} \\
\vdots \\
\mu_{N}
\end{array}\right]
\end{aligned}
$$

where $\mathbf{P}=\left[p_{1}, p_{2}, \cdots, \ldots\right]$ is termed as projection vector; $\mathbf{W}=\left(w_{i j}\right) \in \mathbf{W}^{M} \times \mathbf{W}^{N}$ is named as system matrix, in which $w_{i j}$ describes the contribution of the $i t h$ ray to the $j t h$ pixel, and it can be calculated based on the model shown in figure $4 ; \mathbf{U}=\left[\mu_{1}, \mu_{2}, \ldots \ldots\right.$, is image vector to be reconstructed. 


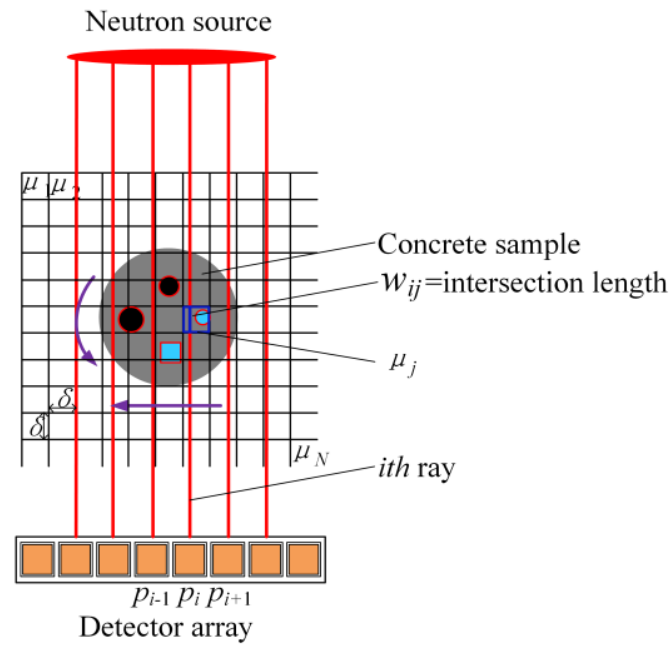

Fig. 4. System matrix calculation model

Usually, $\operatorname{rank}(\mathbf{W})=\operatorname{rank}(\mathbf{W} \mid \mathbf{P})<\mathrm{N}(\mathbf{W}$ and $(\mathbf{W} \mid \mathbf{P})$ are termed as the coefficient matrix and augmented matrix for linear equation set respectively), and equations (2) and (3) are undetermined and ill-posed, thus the exact solution can't be obtained. Therefore, the key point of CT image reconstruction with iteration class algorithms lies in constructing the method to solve the undetermined equation.

In this paper, the sparse algorithm based on total variation (TV) minimization-adaptive steepest descent-projection onto convex sets (ASD-POCS) is employed to implement the image reconstruction, which describes the minimization:

$$
\|\mathbf{P}-\mathbf{W U}\|_{2}^{2}+\alpha\|\mathbf{\Psi}(\mathbf{U})\|_{n}
$$

where $\|\mathbf{P}-\mathbf{W U}\|_{2}^{2}$ is fidelity term; $\|\Psi(\mathbf{U})\|_{n}$ is sparse constrait term; $\|\Delta\|_{n}$ is $l_{n}$-norm of vector $\Delta(\mathrm{n}=0,1$, 2) ; $\alpha$ is sparse constraint coefficient. If $\Psi$ is image gradient transformation, thus :

$$
\|\Psi(\mathbf{U})\|_{1}=\sqrt{\left(\mu_{s, t}-\mu_{s-1, t}\right)^{2}+\left(\mu_{s, t}-\mu_{s-1, t}\right)^{2}}
$$

where $s$ and $t$ are the pixel indexes on the reconstructed image. Equation (5) can also be termed as total variation of image. ASD-POCS algorithm employs simultaneous algebraic reconstruction technique (SART) to solve the minimization of fidelity term, and adaptive steepest descent method to minimize sparse constraint term.

\section{Projection data simulation and reconstruction results}

The configuration of fast neutron $\mathrm{CT}$ is shown in figure 5. In our research, a cylindrical concrete containing two iron bars and two acrylic bars is as the simulation phantom. Since the pixel size of fast neutron detector is
$2 \mathrm{~cm}$, to improve the space resolution on the reconstructed image, the linear scanning method is proposed. In scanning porcess, the concrete sample is rotated and translated step by step in each rotated angle to take the projection data for image reconstruction.

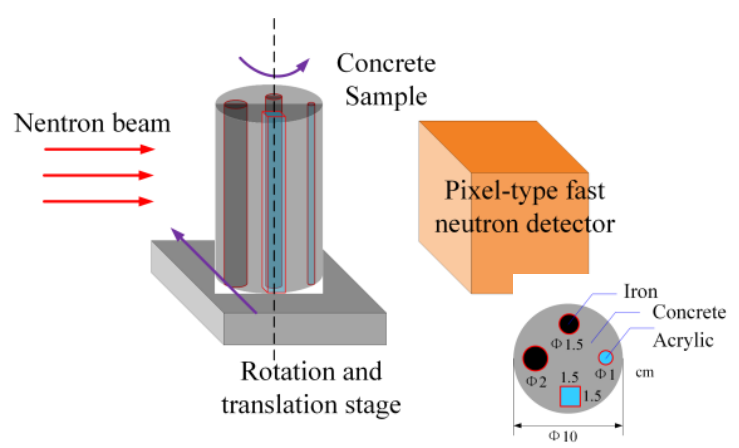

Fig. 5. Fast neutron CT setup

The simulation is implemented by Geant 4 code, and the main parameters are shown in table 1 .

Table 1. Main simulation parameters of fast neutron CT

\begin{tabular}{|l|l|}
\hline Parameter & value \\
\hline Physics & QBBC \\
\hline Neutron spectrum & Shown in figure 2 \\
\hline Record of detector & $\begin{array}{l}\text { Energy deposition of } \\
\text { recoiled proton }\end{array}$ \\
\hline $\begin{array}{l}\text { Number of rotated angle and } \\
\text { step }\end{array}$ & $6 / 30^{\circ}\left(180^{\circ}\right)$ \\
\hline $\begin{array}{l}\text { Translation times and step in } \\
\text { each rotated angle }\end{array}$ & $260 / 1 \mathrm{~mm}$ \\
\hline
\end{tabular}

By means of the simulated radiography images of concrete sample with or without iron and acrylic bars, the projection data for image reconstruction can be obtained. Combing with the system matrix and projection data, the image reconstruction is implemented by ASD-POCS algorithm, and the results is shown in table 2. In the reconstruction, we have changed the translation step size and pixel size on the reconstructed image. It can be observed that the accuracy of reconstructed image basically can be improved as the translation step decreasing, especially in the case that the pixel size on the reconstructed image is $1.25 \mathrm{~mm}$. The reason is that the undermined degree of equation (2) can be improved by increasing the number of projection data, especially in the case that the equation is seriously undetermined. It also can be seen that decrease of pixel size (increase of the number of pixels) on the reconstructed image basically will decrease the accuracy of reconstructed image. That is because increasing the number of unknown number in equation (2) may result in it transiting from overdetermined state to undetermined state. In general, the iron bar with $1.5 \mathrm{~cm}$ and $2 \mathrm{~cm}$ diameters, acrylic bar with $1 \mathrm{~cm}$ diameter and $1.5 \mathrm{~cm}^{2}$ cross section can be identified by the reconstruction after 6 times rotation scanning with $5 \mathrm{~mm}$ translation step in each rotated angle. It demonstrate that the proposed scanning 
method can be applied to fast neutron CT to improve the space resolution of imaging.

Table 2. Reconstructed results under different pixel size on reconstructed image and translation step in each rotated angle

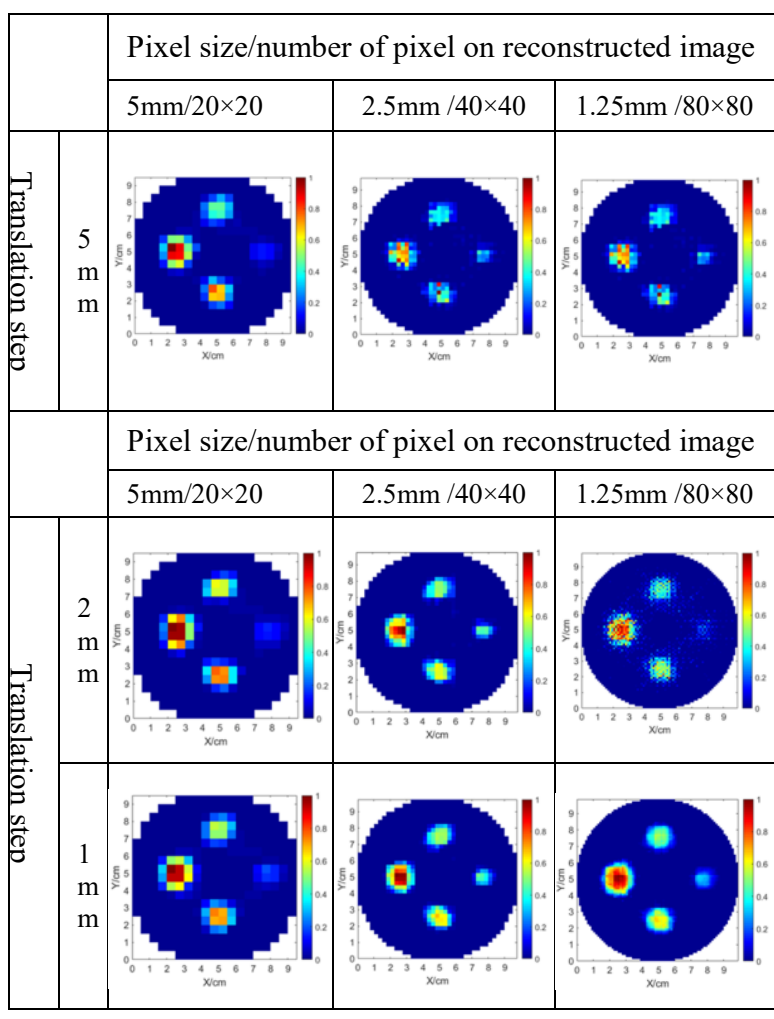

\section{Conclusion and outlook}

This paper has proposed a rotation+linear scanning method for fast neutron imaging with a pixel-type detector. It is shown that iron bar with $1.5 \mathrm{~cm}$ and $2 \mathrm{~cm}$ diameters, acrylic bar with $1 \mathrm{~cm}$ diameter and $1.5 \mathrm{~cm}^{2}$ cross section can be identified by the reconstruction after 6 times rotation scanning with $5 \mathrm{~mm}$ translation step in each rotated angle.
The quantitative evaluation of the accuracy and space resolution of reconstructed image is in progress. And the real experiment will be performed on RANS. We are also planning to employ the area model to calculate the system matrix for the fast neutron CT to get a better space resolution.

This work is supported by a kakenhi Grant-in-Aid for JSPS Fellows (18F18763) from Japan Society for the Promotion of Science.

\section{References}

1. K. U. R. Sardar, Z. Ibrahim, S. A. Memon, M. Jameel, Constr. Build Mater. 107, 58-86 (2016)

2. Y. Otake, Y. Seki, Y. Wakabayashi, Y. Ikeda, T. Hashiguchi, Y. Yoshimura, H. Sunaga, A. Taketani, M. Mizuta, Y. Oshima, M. Ishida, J. Disaster Res. 12, 585-592 (2017)

3. Y. Seki, A. Taketani, T. Hashiguchi, S. Wang, M. Mizuta, Y. Wakabayashi, Y. Otake, Y. Yamagata, H. Baba, K. Kino, K. Hirota, S. Tanaka, Nucl. Instrum. Methods Phys. Res. A 870, 148-155 (2017)

4. R. Zboraya, R. Adamsb, Z. Kis, Appl. Radiat. Isotopes 119, 43-50 (2017)

5. M. F. Yan, H. S. Hu, Y. Otake, A. Taketani, Y. Wakabayashi, S. Yanagimachi, S. Wang, Z. H. Pan, G. Hu, Meas. Sci. Technol. 29, 055404 (2018)

6. S.Agostinelli, J. Allison, K.Amako, J. Apostolakis, H. Araujo, P. Arce, M. Asai, D. Axen, S. Banerjee, et. al, Nucl. Instrum. Methods Phys. Res. A 506, 250-303 (2003)

7. Y. Wakabayashi, A. Taketani, T. Hashiguchi, Y. Ikeda, T. Kobayashi, S. Wang, M. F. Yan, M. Harada, Y. Ikeda, Y. Otake, J. Nucl. Sci. Technol. 55, 859-867 (2018) 